\title{
Être ou ne pas être agriculteur. Capital scolaire et devenir socioprofessionnel des fils d'agriculteurs
}

\author{
Christophe Giraud', Jacques Rémy²
}

\section{Introduction ${ }^{3}$}

Les transformations du système scolaire sur le dernier demi-siècle ont contribué à faire entrer massivement et durablement les enfants de milieux sociaux, comme les agriculteurs ou les ouvriers, qui étaient auparavant relativement exclus de cette institution. Deux étapes doivent être distinguées. Les années 1960 et 1970 ont progressivement conduit à la généralisation et la prolongation de la scolarisation jusqu'au collège. La construction de collèges dans les campagnes, l'allongement de la scolarité obligatoire en 1967, la création et la multiplication de filières techniques ont amené une part accrue des classes populaires à valider un diplôme avant de quitter le système scolaire. L'accès quasi général au collège de tous les enfants d'une même classe d'âge a favorisé l'augmentation des sorties du système scolaire avec le BEPC (brevet d'études du premier cycle du second degré). L'enseignement supérieur et l'accès au lycée dans cette première période de massification scolaire sont restés réservés aux classes moyennes et supérieures (Maurin, 2007). À partir du milieu des années 1980, une deuxième étape initie l'entrée massive des enfants de milieu populaire dans l'enseignement secondaire long (le lycée) et dans l'enseignement supérieur. C'est la "deuxième explosion scolaire" (Chauvel, 2002).

Avec ces réformes, l'injonction scolaire est venue s'imposer aux milieux sociaux jusqu'alors les plus distants de l'école : les ouvriers (Schwartz, 1990 ; Poullaouec, 2004 ) et les agriculteurs. Les fils d'agriculteurs sont ceux qui ont connu la

1. Centre de Recherches sur les liens sociaux (CERLIS - UMR 8070 [Univ. Paris Descartes/ CNRS/ Univ. Paris 3) Univ. Paris Descartes, Centre des Saints-Pères, Bâtiment Jacob, 45, rue des Saints-Pères, 75270 Paris cedex 06, France; INED, UR 3 « Fécondité, famille, sexualité », 45, rue des Saints-Pères, 75270 Paris Cedex 06, France, christophegiraud@me.com

2. INRA, UMR SADAPT, Équipe Proximités - La Racrie 61110 Bellou-sur-Huisne, jacquesremy@numericable.fr

3. Nous remercions nos relecteurs, en particulier Pierre Gasselin, Céline Bessière, Gilles Laferté et Ivan Bruneau.

Ce chapitre de l'ouvrage L'agriculture en famille : travailler, réinventer, transmettre est publié en Open Access sous licence creative commons CC-BY-NC-ND permettant l'utilisation non commerciale, la distribution, la reproduction 
transformation la plus rapide et la plus importante dans leur rapport à l'école (CEuvrard et Rondeau, 1985 ; Davaillon, 1998). La position scolaire des fils d'agriculteurs s'est améliorée davantage que celle du reste de la population. Les sorties sans diplôme étaient beaucoup plus fréquentes pour les fils d'agriculteurs que pour l'ensemble des hommes nés avant 1940. Avec la première massification, la situation s'est progressivement inversée puisque 14,4\% seulement des fils d'agriculteurs nés entre 1960 et 1969 sont sortis sans diplôme ou avec le seul Certificat d'études primaires (CEP) (23,3\% pour les fils d'ouvriers) ${ }^{4}$. Les enfants d'agriculteurs ont acquis un niveau de capital scolaire supérieur à celui des enfants d'ouvriers puisque leur scolarité est moins marquée par le redoublement (Euvrard et Rondeau, 1985).

Les fils d'agriculteurs s'orientent plus souvent vers des filières techniques ou professionnelles. Ce goût pour les filières techniques longues se manifeste également dans le développement récent des formations de niveau BTSA ${ }^{5}$ dans les établissements agricoles dont les effectifs ont presque triplé de 1976 à 1986 (Hermelin-Guillou, 1987). Cette orientation vers l'enseignement technique n'est pas un choix contraint par une scolarité " chaotique ». Toutefois, la diversité des formations agricoles offertes (comme celles, proposées par les Maisons familiales rurales, qui se font « en alternance ") peut permettre à certains enfants réfractaires à la logique scolaire de poursuivre leur scolarité (Alarcon, 2008).

La première massification scolaire, à partir de 1959, a socialisé plus longtemps les enfants à la culture scolaire. Les diplômes, même de niveau modeste, ont constitué une ressource importante pour le " désenclavement " culturel des classes populaires (et donc d'une partie des agriculteurs), pour penser leur " participation aux formes symboliques dominantes" (Schwartz, 2011). L'école a aussi été analysée comme un facteur d'éloignement du milieu agricole qui a favorisé l'entrée dans le salariat, principalement ouvrier (Jégouzo et Brangeon, 1976). Elle aurait donc contribué très fortement à la réduction de la population agricole et à la crise de la reproduction agricole (Champagne, 2002).

Cette généralisation de l'accès au collège puis à l'enseignement secondaire long a pris une forme particulière dans les mondes agricoles. Les lois d'orientation des années 1960 et 1962 ont donné son autonomie à l'enseignement agricole ; il échappait ainsi à la tutelle de l'Éducation nationale tout en adossant ses formations aux enseignements techniques dispensés dans les collèges et les lycées. L'école et ses titres scolaires sont devenus alors des instruments de sélection et de socialisation professionnelle. Toute une hiérarchie de formations et de diplômes, négociée avec la profession, s'est progressivement mise en place dans les années 1960-1970 dans des établissements spécifiques (Hermelin-Guillou, 1987). À partir de 1974, dans le cadre de la politique agricole de « cogestion » conduite par l'État et la profession

4. Sans mention d'une autre référence, tous les chiffres de cette introduction proviennent de l'enquête "Étude de l'histoire familiale de 1999 " réalisée par l'Insee et l'Ined, calculs personnels. 5. Brevet de technicien supérieur agricole, diplôme de niveau III, équivalent d'un BTS, dans la classification interministérielle des qualifications professionnelles qui va de V (niveau le plus faible) à I (niveau le plus élevé). 
(Coulomb, 1990), un dispositif de sélection par l'élévation progressive du niveau scolaire exigé des candidats aux aides s'est mis en place. Désormais, il faut disposer d'un titre scolaire pour pouvoir s'installer en agriculture en bénéficiant d'aides financières ${ }^{6}$.

Tout à la fois instrument de dépaysannisation et de professionnalisation, l'école a donc des effets divergents sur le devenir socioprofessionnel des fils d'agriculteurs : trop d'école peut éloigner les enfants d'agriculteurs de ce milieu, et pas assez obère les chances d'installation dans les meilleures conditions. Notre hypothèse est que le capital scolaire technique (agricole) est devenu, pour ces générations de la première massification scolaire et du conditionnement des aides à l'installation, une condition centrale du maintien des fils d'agriculteurs dans la profession agricole et que la possession de ces capitaux scolaires recompose les hiérarchies sociales qui pouvaient exister au sein des groupes sociaux agricoles. L'absence de capital scolaire ou la possession d'un capital scolaire non technique a en revanche éloigné les fils d'agriculteurs du milieu agricole et les a dirigés vers des positions de l'espace social différentes selon leurs ressources scolaires.

C'est dans ce contexte institutionnel de la première massification scolaire et de la politique de conditionnement des aides à l'installation et dans la ligne de plusieurs travaux récents (Alarcon, 2008 ; Bessière, 2010) que nous montrerons à partir d'enquêtes statistiques (Encadré 1) d'abord les facteurs qui expliquent comment les agriculteurs de cette génération de la première massification scolaire ont été conduits au cours des années 1960-1970 à développer ce nouveau rapport positif au système scolaire ( $1^{\text {re }}$ partie de ce chapitre). Puis nous examinerons comment le capital scolaire acquis avec cette première massification pèse sur les trajectoires socioprofessionnelles des fils devenus agriculteurs ( $2^{\mathrm{e}}$ partie), comme sur celles des fils qui ne le sont pas devenus ( $3^{\mathrm{e}}$ partie). Si bien d'autres facteurs entrent en jeu dans les transformations que l'on observe, comme l'effritement régulier de la composante familiale des collectifs de travail des exploitations agricoles, les profondes évolutions de la scolarité et du rapport à l'école des familles agricoles et de leurs enfants offrent des clés de compréhension des choix et contraintes auxquels se trouvent confrontés les uns et les autres. Tel est le sens de notre présente contribution à l'analyse des recompositions au sein des exploitations agricoles.

6. L'installation sans aide pouvait par ailleurs toujours se faire sans la possession d'aucun diplôme. 
Encadré 1. Sources et utilisation des données.

Notre analyse s'appuie essentiellement sur deux enquêtes Insee : l'enquête Étude de l'histoire familiale $(E H F)$ réalisée par l'Insee (Institut national de la statistique et des études économiques) en 1999 sur un échantillon de près de 380000 individus de plus de 18 ans. Elle portait sur des thématiques relatives à la vie familiale et à la vie professionnelle. 19587 fils d'agriculteurs ont été interrogés. Nous avons également eu recours à l'enquête Formation et qualification professionnelle (FQP) réalisée en 2003 auprès d'un échantillon de presque 40000 personnes de plus de 15 ans dont 2348 fils d'un père ou d'une mère agriculteur. L'enquête avait pour cœur la formation professionnelle et le parcours professionnel des individus interrogés, de leur conjoint et de leurs ascendants. Ces données sont les plus récentes disponibles au moment de la rédaction de ce texte.

De façon très ponctuelle, les recensements agricoles 2000 et 2010 ont été utilisés pour compléter les résultats. Ils ne constituent cependant qu'une source de données accessoire ici car les recensements ne sont pas des enquêtes de mobilité sociale et que leur population n'est pas exactement la même que celle des enquêtes Insee. Rappelons que les données établies par l'Insee appréhendent des individus qui se sont déclarés agriculteurs au recensement de la population, tandis que les statistiques du SSP (Service de la statistique et de la prospective du ministère de l'Agriculture) recouvrent l'ensemble des exploitants agricoles, nombre d'entre eux n'étant pas agriculteurs à titre principal (ni même à titre secondaire pour une part d'entre eux) (Rémy, 2012 : 362-363).

\section{La population des fils d'agriculteurs}

Notre analyse de la relation entre capital scolaire et trajectoires socioprofessionnelles se limite aux fils d'agriculteurs. La question de l'accès à la profession d'agriculteur (trice) se pose en des termes très différents pour les hommes et pour les femmes de la génération étudiée. Pour les hommes le choix dépend fortement du diplôme détenu et de la façon dont les parents ont préparé un successeur. Les femmes sont le plus souvent écartées de cette transmission familiale du métier et de la perspective de succéder (Bessière, 2010). Elles sont ainsi moins fréquemment héritières du capital productif qui leur permettrait de s'installer (Barthez, 1994). Devenir agricultrice est conditionné, dans cette génération, le plus souvent par un choix conjugal bien plus que par une formation scolaire (Giraud, 2014), même si les femmes passent aussi et de plus en plus par la formation agricole (Dahache, 2013).

Une analyse par classe d'âge avec une attention centrale pour les fils ayant connu la première massification scolaire

En raison des changements profonds et rapides au sein du système éducatif français depuis cinquante ans, l'analyse du rapport à l'école et des effets du diplôme imposait de diviser notre population de fils en classes d'âge. 
Pour l'analyse de l'insertion professionnelle, nous avons privilégié la classe des individus âgés de 40 à 49 ans en 1999 (hommes nés entre 1950 et 1959) qui a été la première à connaître l'allongement de la scolarité obligatoire et l'accès aux études secondaires ainsi que les premières mesures de conditionnement scolaire des aides à l'installation. C'est une classe d'âge où les carrières sont bien stabilisées et où les installations, parfois tardives en agriculture, sont réalisées. Nous avons comparé cette cohorte avec celle des individus âgés de 30 à 39 ans (hommes nés entre 1960 et 1969), qui a connu pour partie l'instauration du collège unique, la montée des filières techniques courtes et a été complètement soumise à la politique sélective en matière d'installation. La cohorte la plus jeune des moins de 30 ans à la date de l'enquête a connu la "seconde explosion scolaire " mais est composée de personnes qui n'ont pas forcément fini leur scolarité et qui n'ont pas une position professionnelle stabilisée. Nous l'avons donc écartée de notre analyse des trajectoires socioprofessionnelles. L'étude du devenir socioprofessionnel des enfants de cette seconde explosion scolaire reste à faire.

\section{Les facteurs de variation dans l'appropriation du capital scolaire par les agriculteurs}

Le milieu social d'origine est un facteur central qui explique une partie des variations dans l'acquisition du capital scolaire au sein de la génération des enfants de la première (comme de la seconde) massification scolaire. En effet, des différences sociales considérables traversent le monde agricole, en termes de place dans l'échelle sociale approchée par le capital économique possédé comme en termes de plus ou moins grande ouverture culturelle de la famille.

\section{Position sociale au sein de l'agriculture et acquisition du capital scolaire}

Le capital économique de l'exploitation des parents, approché par la distinction entre agriculteurs sur petite, moyenne et grande exploitation, établie par l'Insee ${ }^{7}$, est un premier facteur qui intervient dans l'acquisition d'un capital scolaire élevé, que celui-ci soit de nature générale ou technique. La dimension économique de l'exploitation parentale facilite ou freine l'acquisition de capital scolaire des fils.

7. Selon l'Insee les exploitations sont distinguées selon leur dimension économique, à partir d'un indicateur qui mêle les surfaces utilisées et l'orientation productive des exploitations. La dimension économique est mesurée sur la base d'une échelle dont l'unité est "l'hectare équivalent blé ". Les petites exploitations sont celles dont la dimension économique est inférieure à 20 hectares équivalent blé, les exploitations moyennes se situent entre 20 et moins de 40 hectares équivalent blé et les grandes exploitations sont supérieures ou égales à 40 hectares équivalent blé. 
D'après l'enquête FQP (2003), les fils nés entre 1950 et 1959 et entre 1960 et 1969 de petits agriculteurs ont ainsi deux fois plus tendance que les fils d'agriculteurs sur moyenne ou grande exploitation à quitter le système d'enseignement sans aucun diplôme (Fig. 1). Ces derniers accèdent plus aisément à un niveau d'enseignement général supérieur ou égal au bac. Le niveau monte, les écarts restent.

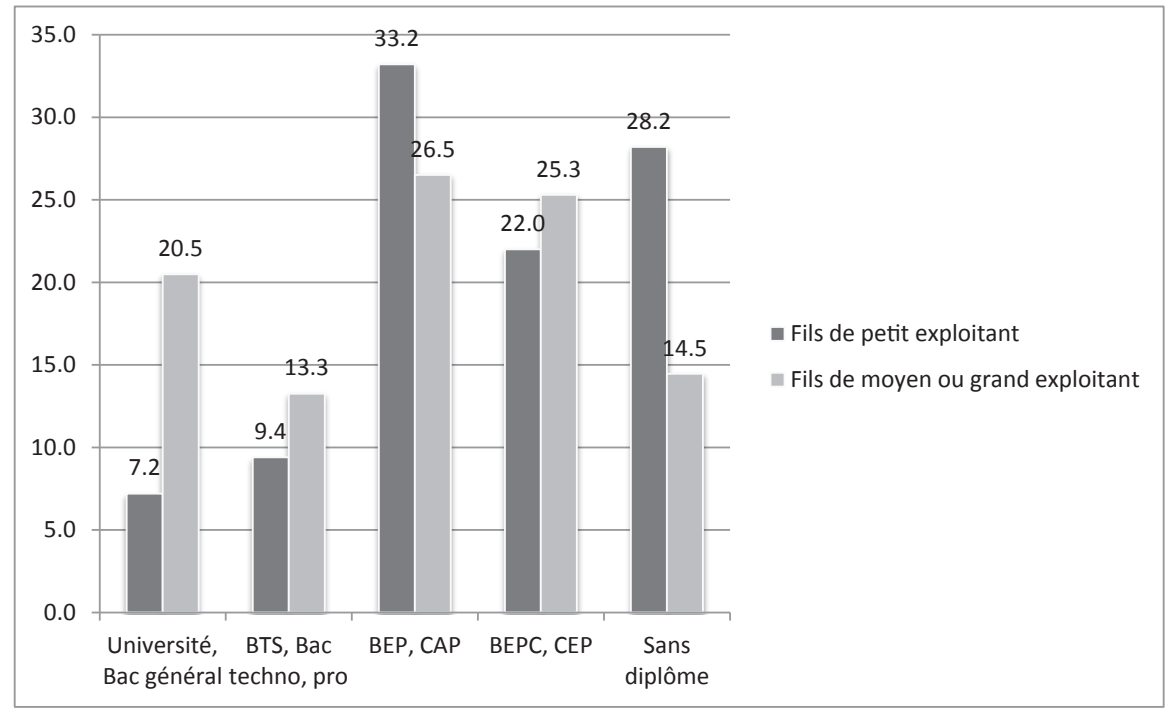

Source : FQP2003.

Champ : fils d'agriculteur nés entre 1950 et 1959, âgés de 40 à 49 ans (N=583) en 1999.

Lecture : 7,2\% des fils de petit exploitant àgés de 40 à 49 ans ont obtenu le bac général ou un diplôme universitaire.

Test du Khi2 : Significatif au seuil de 0,03\%.

Fig. 1. Niveau de diplôme des fils d'agriculteurs selon la dimension économique de l'exploitation du père.

L'influence du milieu social d'origine ne réside pas seulement en des différences d'aide financière, elle repose aussi sur une différence culturelle : le milieu des grandes exploitations est plus ouvert à la certification scolaire des compétences (on y reviendra dans la $2^{\mathrm{e}}$ partie). Ainsi, les familles des grandes exploitations viticoles disposent d'une culture plus proche de la culture scolaire que celles des petites exploitations car les compétences scolaires avancées peuvent être utiles au sein de ces exploitations aux domaines d'activité variés (Bessière, 2010).

Il ne faut pas surestimer cependant la proximité des catégories sociales agricoles aisées avec la culture légitime : si celles-ci ont plus de chance que les petits agriculteurs de donner à leurs enfants un capital scolaire élevé, une part importante des fils quitte le système scolaire avec un bagage léger. 14,5\% des fils âgés de 40 à 49 ans des familles de grand ou moyen exploitants n'ont aucun diplôme (28,2 \% des fils de 
petits exploitants sont dans ce cas), soit un peu plus souvent que les fils dont le père exerce une profession intermédiaire $(9,6 \%)$ ou que les fils dont le père est cadre ou d'une profession intellectuelles supérieure $(7,9 \%)$. Ils font cependant mieux que les fils d'employés (16,3\%), que les fils d'indépendants (artisans, commerçants, chefs d'entreprise) ou que les fils d'ouvriers hors agriculture $(27,7 \%)$. Si l'on observe les chances d'acquérir un diplôme élevé (équivalent bac ou plus) alors les hiérarchies sont similaires : les fils de moyens ou grands exploitants sont au même niveau que les fils d'employés (20,5\% contre 20,3\%), moins avantagés que les enfants issus des professions intermédiaires $(25,9 \%)$ et très loin des fils de cadres et professions intellectuelles ( $53 \%$ ) mais font beaucoup mieux que les fils d'ouvriers $(6,8 \%)$. La proximité avec la culture légitime est donc toute relative : les fils de grands exploitants sont plus proches de la culture des classes moyennes que de la culture ouvrière.

Le risque pour les fils de petits agriculteurs de quitter l'école sans diplôme est très proche de celui qu'encourent les ouvriers hors agriculture $(28,2 \%$ contre $27,7 \%)$; il demeure très inférieur à celui des fils d'ouvriers agricoles (45,2\%). Les chances d'acquérir un diplôme de niveau bac et plus des fils de petits exploitants sont similaires à celles des fils d'ouvriers qualifiés (7,2 \% contre $8 \%$ ), mais restent loin des fils d'employés (20,3\%). Leur rapport à la culture reste proche des couches qualifiées du monde ouvrier. Les avancées scolaires observées pendant les années 1960 et 1970 par Euvrard et Rondeau touchent bien toutes les strates de l'agriculture mais sont plus marquées encore chez les fils des fractions aisées de l'agriculture dont le rapport à la culture scolaire est assez éloigné de celui des fractions défavorisées qui conservent des similarités fortes avec le monde ouvrier.

\section{L'ouverture culturelle des parents}

La composition sociale du couple parental constitue également un facteur d'ouverture ou de fermeture par rapport à la culture scolaire. Depuis les années 1960, la situation d'hétérogamie professionnelle et sociale n'a cessé de croître en agriculture pour devenir la situation dominante aujourd'hui chez les jeunes agriculteurs (Giraud et Rémy, 2008). La transformation des systèmes de production avec l'augmentation de la population des exploitations en grandes cultures et l'affaiblissement des exploitations peu spécialisées (en polyculture-élevage) a conduit à une déconjugalisation du travail agricole ; les conjointes des exploitants spécialisés exercent le plus souvent une autre activité professionnelle qu'elles conservent après l'entrée en couple (Dufour et Giraud, 2012). Or, avoir un père agriculteur et une mère salariée dans le secteur des services conduit à mettre en doute chez les enfants l'évidence que l'univers agricole est le seul valable, le seul possible. Il introduit des éléments de comparaison concrets qui permettent aux enfants de se projeter dans d'autres univers professionnels. Le conjoint salarié, comme les collatéraux devenus salariés hors agriculture (Champagne, 2002), remettent en cause l'évidence du mode de vie et de la profession agricole. 
L'effet de la composition du couple parental doit être pensé en interaction avec le niveau de capital économique de l'exploitation agricole. Nous avons donc construit une variable synthétique qui permet de distinguer les différentes situations familiales et agricoles (Fig. 2).

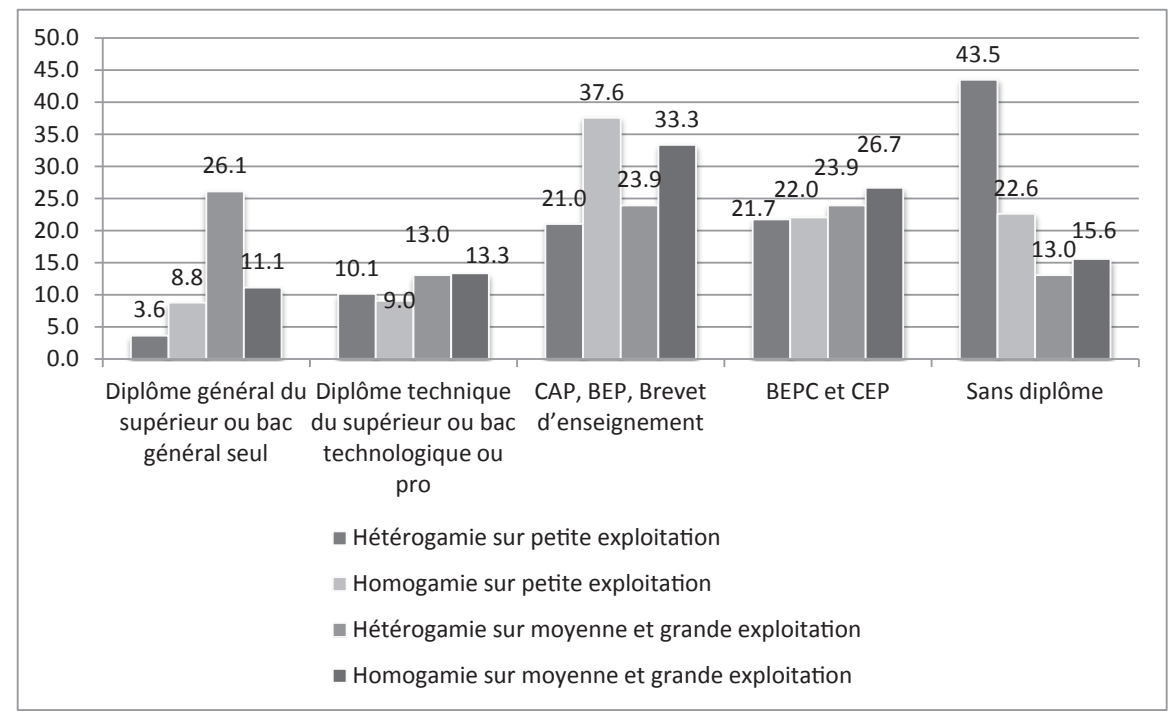

Source : FQP2003.

Champ : hommes àgés de 40 à 49 ans en 1999 ayant un père exploitant $(N=583)^{8}$.

Lecture: 5, $6 \%$ des hommes dont l'un des parents seulement était agriculteur sur une petite exploitation agricole ont obtenu un diplôme équivalent ou supérieur au bac général.

Test du Khi2 : significatif au seuil de 0,01\%.

Fig. 2. Niveau de diplôme des fils d'agriculteurs de 40 à 49 ans selon I'homogamie parentale et la dimension économique de l'exploitation des parents

Pour la génération des fils nés entre 1950 et 1959, être un fils issu d'une famille homogame (quelle que soit la taille de l'exploitation parentale) renforce les chances d'acquérir un diplôme technique $\left(\mathrm{CAP}^{9}, \mathrm{BEP}^{10}\right.$, brevet professionnel $\left.{ }^{11}\right)$. À l'inverse, être issu d'une famille hétérogame détourne relativement des diplômes techniques : pour les exploitations les plus importantes, les fils ont plus tendance que les autres à se tourner vers des diplômes généraux élevés $(26,1 \%$ ont un diplôme universitaire

8. En raison de faibles effectifs pour la génération des trentenaires, nous n'analysons que la classe d'âge des 40-49 ans.

9. Certificat d'aptitude professionnelle, diplôme de niveau V.

10. Brevet d'études professionnelles, diplôme de niveau $\mathrm{V}$.

11. Diplôme de niveau IV. 
ou le bac contre 3,6 \% pour les fils sur petite exploitation) ${ }^{12}$. Être issu d'une famille hétérogame et d'une petite exploitation dans cette génération condamne en revanche plus souvent les fils à une sortie précoce du système scolaire $(43,5 \%$ sortent sans diplôme, soit le double des fils d'une famille agricole homogame travaillant sur une petite exploitation) : la faiblesse du capital économique est un obstacle à l'acquisition du capital scolaire mais le fait d'avoir des parents ayant des métiers différents ajoute un effet négatif supplémentaire. En effet, le recrutement social des conjoints est très différent dans ce cas-là : les couples dans les petites exploitations hétérogames, à la différence des moyennes et grandes exploitations hétérogames sont plus souvent composés d'un agriculteur et d'une conjointe ouvrière ou employée d'exécution, disposant de peu de capitaux scolaire et sociaux à transmettre (Giraud, 2014).

La composition du couple parental influence fortement la structure du capital scolaire (plus ou moins orienté vers des diplômes généraux ou techniques). Avoir obtenu un diplôme technique pour cette génération est plus souvent le fait de fils dont le monde parental est exclusivement agricole. L’hétérogamie parentale conduit à une ouverture du monde social et favorise par suite une orientation vers des cursus généraux ou vers la sortie du système scolaire sans diplôme.

La première explosion scolaire a conduit à l'accroissement global du capital scolaire des fils d'agriculteurs. Ceux-ci ont acquis un capital scolaire technique plus élevé que les fils d'ouvriers de la même génération. Mais cette augmentation généralisée du capital scolaire est restée marquée par les inégalités qui existent à l'intérieur du milieu agricole. Les inégalités dans le rapport à la culture légitime sont produites par la position des familles dans les différents milieux sociaux de l'agriculture et par la composition sociale des familles. Nous nous tournons maintenant vers l'analyse du poids du capital scolaire sur les trajectoires socioprofessionnelles des fils qui ont connu la première massification scolaire. Nous distinguerons les fils qui sont devenus agriculteur de ceux qui se sont engagés dans une autre profession.

\section{Devenir agriculteur : des positions sociales scolairement clivées}

Sur la période considérée, le fait majeur est celui de l'élimination des plus petites exploitations. La part des fils d'agriculteurs qui deviennent agriculteurs connaît donc une baisse continue sur cinquante ans (Tab. 1).

12. Il faudrait pouvoir distinguer finement les niveaux CAP et BEP, ce que l'enquête FQP2003 n'autorise pas. L'enquête EHF, quant à elle, ne permet pas de distinguer la taille de l'exploitation dont les fils sont issus. 
Tab. 1. Part des fils d'agriculteurs âgés de 40 à 49 ans étant devenus agriculteurs.

\begin{tabular}{|c|c|c|c|c|}
\hline & $1953^{13}$ & 1970 & 1977 & 2003 \\
\hline $\begin{array}{l}\text { Agriculteurs, } \\
\text { fils d'agriculteurs }\end{array}$ & $60 \%$ & $42 \%$ & $38 \%$ & $29 \%$ \\
\hline
\end{tabular}

Sources: enquête sur l'emploi de 1953, enquêtes FQP de 1970, 1977 (Champagne, 2002 : 223) et 2003 (calculs personnels).

Champ: hommes actifs occupés, àgés de 40 à 49 ans, France entière.

Lecture : $60 \%$ des fils d'agriculteurs âgés de 40 à 49 ans en 1953 sont agriculteurs.

La chute du nombre d'exploitations agricoles sur cette période a coïncidé avec le prolongement de la scolarité et l'augmentation du capital scolaire chez les fils d'agriculteurs, semblant valider la thèse de la dépaysannisation par l'école. Nous allons montrer cependant combien le capital scolaire a un rôle central pour l'orientation des fils vers la profession d'agriculteur, comme pour l'accès aux positions élevées à l'intérieur de ce groupe socioprofessionnel.

\section{Le temps des diplômés}

Deux populations se dessinent parmi les fils d'agriculteurs qui ont connu la première massification scolaire et qui sont devenus eux-mêmes agriculteurs (Fig. 3) : une population disposant d'un fort capital technique (de niveau BEP ou bac technique ou professionnel) et une population qui n'a pu décrocher de titre scolaire technique (disposant au mieux du BEPC). Ces deux populations sont dans des dynamiques de reproduction très différentes : les diplômés voient leur capacité à choisir l'agriculture demeurer très élevée chez les agriculteurs âgés de 30 à 39 ans en 1999. Par contre la capacité des 30-39 ans à faible niveau scolaire à s'installer comme agriculteur se réduit par rapport aux 40-49 ans. La possession d'un diplôme technique se fait de plus en plus indispensable et la part des individus fortement diplômés tend à s'accroitre dans la population des chefs d'exploitation de 2000 à $2010^{14}$ (SSP, 2011 : 11). Contrairement à la période qui a précédé la première explosion scolaire, le diplôme ici ne joue pas contre l'installation en agriculture. Bien au contraire il est presque devenu une condition pour reprendre une exploitation agricole dans la mesure où il est indispensable à l'obtention des aides à l'installation et qu'il facilite l'entrée dans un métier en transformation.

13. Il faut prendre avec précaution les données pour l'année 1953 où la nomenclature des CSP (catégories socio-professionnelles) et les procédures de recueil et de classement ne sont pas encore tout à fait stabilisées.

14. $34 \%$ des chefs d'exploitation de moins de 40 ans sont issus de l'enseignement supérieur contre $18 \%$ en 2000 . 


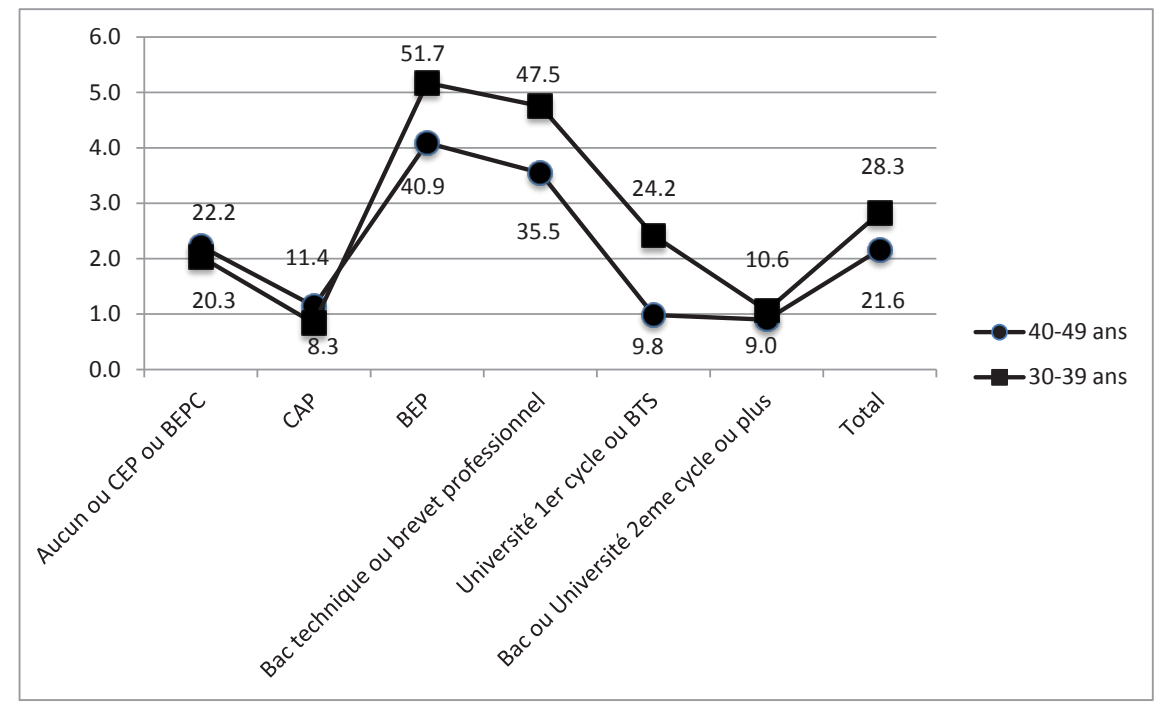

(*) La somme en ligne des pourcentages ne fait pas $100 \%$ car une modalité regroupant certains retraités et inactifs, assez peu fréquente, n'a pas été reproduite dans ce tableau.

Source : EHF1999.

Champ : hommes âgés de 30 à 39 ans et 40 à 49 ans ayant un père agriculteur $(N=7686)$.

Lecture : 22,2 \% des fils d'agriculteurs âgés de 40 à 49 ans dotés au plus d'un BEPC sont agriculteurs.

Fig. 3. Effet du diplôme des fils d'agriculteurs sur les chances d'être agriculteur (en \%).

L'accroissement du capital scolaire technique suit en partie les évolutions législatives (Encadré 2) qui ont conditionné l'obtention des aides à l'installation (Maresca, 1988).

Encadré 2. La politique de conditionnement des aides aux titres scolaires.

La DJA (dotation jeune agriculteur, créée en 1973 pour les zones de montagne et étendue à tout le pays en 1976) et les prêts bonifiés sont versés aux jeunes agriculteurs qui s'installent (pendant cinq ans au moins) à condition de posséder un niveau de formation minimal :

- le BEPA (brevet d'enseignement professionnel agricole, diplôme de niveau $\mathrm{V}$, de même niveau que le BEP) à partir de 1974 (les générations nées entre 1950 et 1959 et surtout entre 1960 et 1969 connaissent ainsi un boom des diplômes de niveau BEP dont l'importance double puis triple par rapport à la génération née entre 1940 et 1949);

- le BTA (brevet de technicien agricole, diplôme de niveau IV, comme le bac), à partir de 1992 ;

- depuis 1996, un diplôme équivalent au bac professionnel (option conduite et gestion de l'exploitation) ou au brevet de technicien agricole, assorti d'une formation professionnelle complémentaire si les personnes qui s'installent sont nées avant 1971. 
Pour la génération née entre 1950 et 1959 (âgées de 40 à 49 ans en 1999), la propension à obtenir un BEP (niveau minimal pour accéder aux aides dès 1974) double par rapport aux générations de la décennie précédente (Fig. 4). Le conditionnement des aides à l'installation au niveau BEP dès 1974 entraîne le déclin du niveau CAP en agriculture et des niveaux de diplôme inférieurs (qui ne concernent plus que $12,8 \%$ de la population). Enfin, pour la génération née après 1970 (qui a 20 ans au cours des années 1990 au moment du nouveau changement de législation), c'est le boom des formations de niveau bac pro, devenant par là même le diplôme le plus fréquent. La détention du BEP reste cependant importante : elle concerne $30 \%$ des agriculteurs, et ouvre, par le biais de stages professionnels, l'accès aux aides).

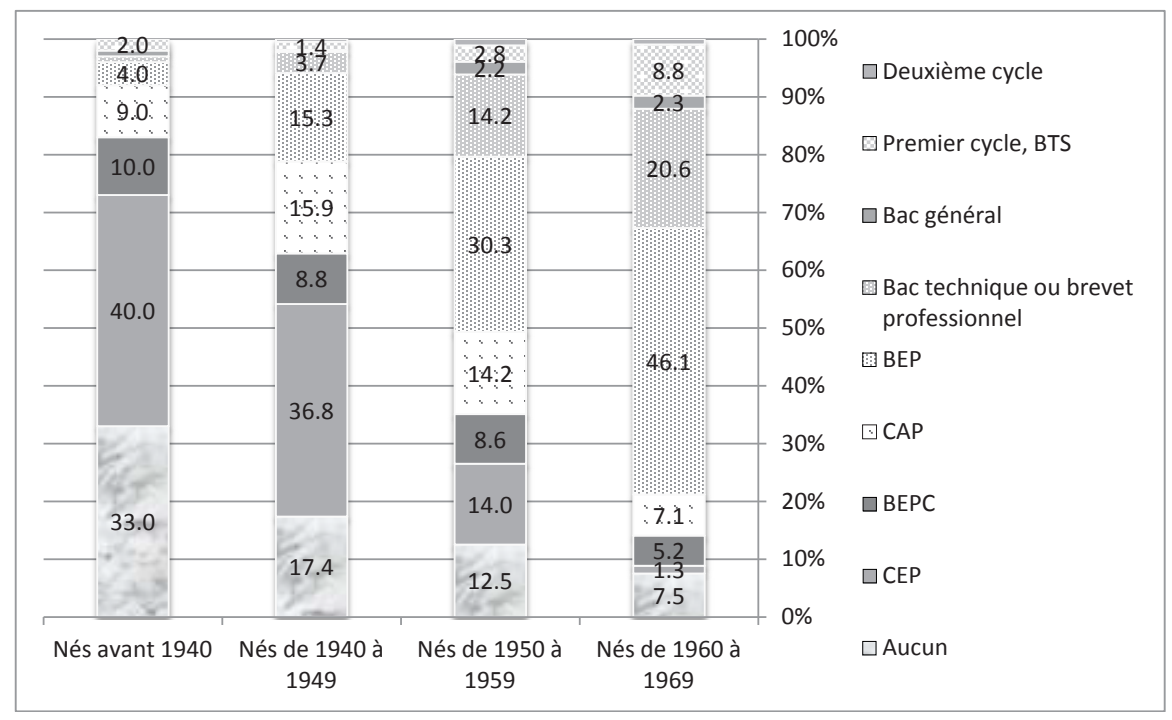

* Les valeurs qui ne dépassent pas $1 \%$ n'ont pas été reproduites ici. Source : EHF1999.

Champ : hommes, fils d'agriculteurs, agriculteurs en activité $(N=2538)$.

Lecture : $33 \%$ des fils d'agriculteurs nés avant 1940 et agriculteurs aujourd'hui ont quitté le système scolaire sans diplôme.

\section{Fig. 4. Profil scolaire des hommes agriculteurs par classe d'âge en 1999*.}

Le capital scolaire des jeunes agriculteurs cependant va au-delà des exigences administratives : presque $20 \%$ des plus jeunes d'entre eux disposent d'un niveau BTS ou premier cycle universitaire en 1999. Cela témoigne de l'intérêt de certains agriculteurs pour l'excellence technique et commerciale, au-delà de l'accès à la DJA.

La transformation du rapport à l'école s'inscrit également dans les transformations du métier d'agriculteur. L'agriculture acquiert une dimension gestionnaire et bureaucratique avec la multiplication des formulaires à remplir pour l'obtention d'aides diverses et des subventions de la Politique agricole commune (PAC). Plus le 
diplôme est élevé et plus la capacité de gestion de l'entreprise et de construction de plans de financement paraît crédible et ouvre sur des coopérations confiantes avec les institutions bancaires. Un bon niveau de formation permet également de travailler à l'extérieur en position d'attente pour une reprise au moment de la retraite des parents (Alarcon, 2008).

\section{L'élan économique des diplômés et le poids de la transmission familiale}

Pour les fils d'agriculteurs devenus agriculteurs, le clivage scolaire renforce un clivage social et économique dans la mesure où les diplômés ont plus souvent reçu une DJA ou bénéficié d'aides financières de type Plan d'amélioration matérielle que les non-diplômés. Les premiers se trouvent donc dans une dynamique économique très différente des seconds car ils sont également les lauréats du système d'encadrement agricole. Le diplôme fonctionne bien comme un capital qui peut être utilisé sur la scène professionnelle pour renforcer sa position au sein du monde agricole.

Cet avantage économique des diplômés est net chez les agriculteurs trentenaires et quarantenaires (Fig. 5) : les agriculteurs, fils d'agriculteurs, dans la quarantaine en 1999, titulaires au moins d'un BEP sont plus souvent exploitants sur grande exploitation que sur moyenne ou petite exploitation. Ce clivage scolaire et économique reproduit fortement les clivages sociaux présents à la génération précédente et le poids de la transmission familiale. Les milieux aisés de l'agriculture en favorisant les études renforcent leur position et l'accroissement de leurs capitaux économiques ${ }^{15}$. Les fils de petits exploitants ont pu en revanche connaître des difficultés matérielles et culturelles qui les ont freinés dans l'acquisition du capital scolaire. Ceux qui ont choisi de rester dans l'agriculture ont dû alors continuer à le faire sans aide, et donc sans incitation économique.

La détention du capital scolaire vient cependant perturber les mécanismes de la transmission familiale : l'élan économique des diplômés est particulièrement net quand on s'intéresse aux agriculteurs qui viennent de la petite agriculture ${ }^{16}$. Pour la génération des fils d'exploitants sur petite exploitation, âgés de 40 à 49 ans, et ayant choisi l'agriculture, les chances de devenir exploitants sur grande exploitation passent de $45,5 \%$ pour les fils pourvus au mieux d'un BEPC à $68,2 \%$ pour les autres diplômés ${ }^{17}$. À dimension économique égale de l'exploitation parentale, disposer d'un diplôme donne des avantages économiques importants pour l'accès aux

15. Les classements de l'enquête FQP ou EHF (agriculteurs sur petite, moyenne ou grande exploitation) rendent difficiles une vérification statistique de ce type de processus pour les enfants des grandes et très grandes exploitations.

16. L'enquête EHF ne distingue pas les fils d'agriculteurs dont le père travaillait sur petite, moyenne ou grande exploitation. Seule l'enquête FQP nous permet d'effectuer ce type de croisement. Mais la faiblesse des effectifs dans cette enquête ne nous permet pas de faire tous les croisements que nous souhaiterions.

17. Comparaison réalisée sur 121 individus. Différence significative au seuil de 1,2 \%. 
fractions élevées de l'agriculture. L'effet du diplôme n'est donc pas que le reflet du poids des capitaux économiques. Le diplôme favorise une certaine mobilité sociale au sein même des groupes sociaux agricoles. Le poids de la composition du couple parental joue ici à plein. Les agriculteurs dont les deux parents sont eux-mêmes agriculteurs ont été éduqués plus souvent comme des successeurs et conduits à rechercher les diplômes ad hoc pour rester dans l'agriculture comme on l'a vu dans la première partie. De même que le niveau scolaire monte au fil des générations, dans l'agriculture la taille des exploitations croît, mais la dynamique de développement est bien différente entre les diplômés et les non diplômés.

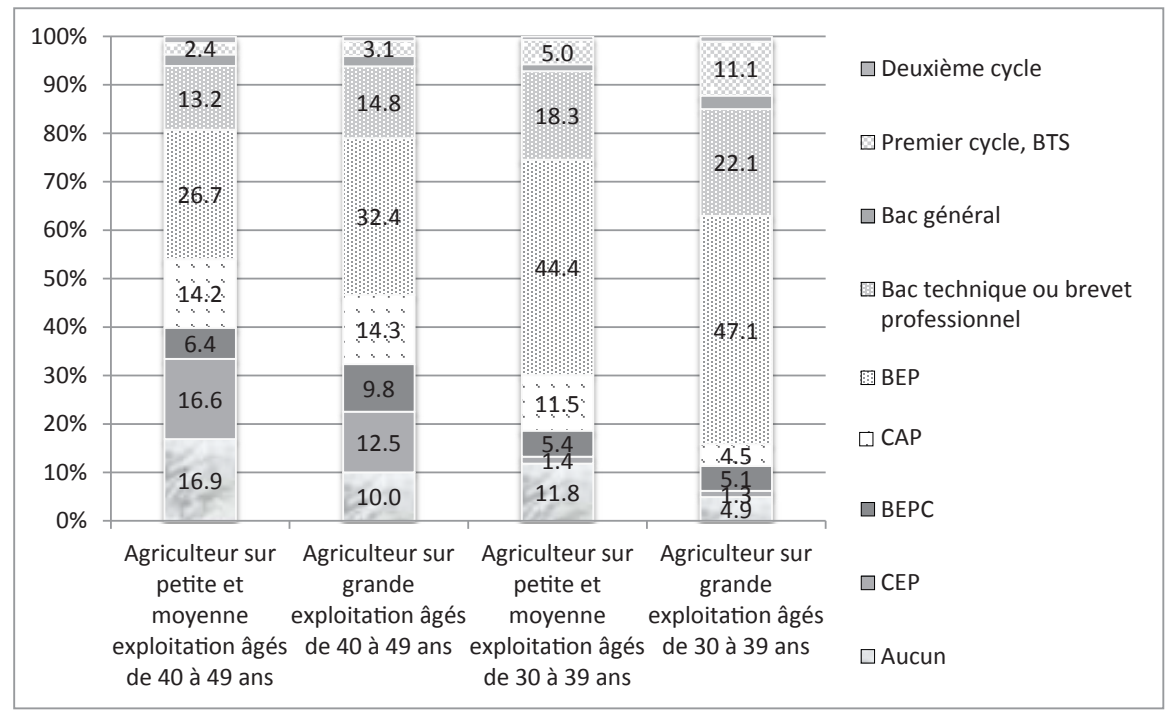

* Les valeurs qui ne dépassent pas $1 \%$ n’ont pas été reproduites ici.

Source : EHF1999.

Champ: hommes, fils d'agriculteurs, agriculteurs àgés de 40 à 49 ans en activité $(N=815)$

Lecture : 16,9\% des fils d'agriculteurs àgés de 40 à 49 ans, eux-mêmes exploitants sur petite et moyenne exploitation ont quitté le système scolaire sans diplôme.

Fig. 5. Profil scolaire des agriculteurs de 30 à 39 ans et de 40 à 49 ans selon la dimension économique de l'exploitation*.

Diplôme, position sociale d'origine dans l'agriculture et composition sociale du couple parental se combinent donc pour déterminer non seulement le maintien ou non des fils dans l'agriculture mais encore pour définir la position occupée par les fils au sein du groupe social des agriculteurs d'aujourd'hui. Au sein de la population agricole actuelle, un nouveau clivage apparaît entre les diplômés et les non-diplômés qui ne recoupe qu'en partie les différences économiques et sociales déjà manifestes dans la génération de leurs parents. 
Témoin de ce nouveau clivage social, de cette nouvelle hiérarchie au sein du groupe social agricole, les choix des conjoints montrent combien les diplômés sont situés plus haut dans l'univers social que les non-diplômés (Giraud et Rémy, 2008).

\section{Des nouvelles hiérarchies au sein de l'agriculture}

L'accroissement du capital scolaire au sein des différentes strates de l'agriculture a conduit à une segmentation supplémentaire du monde agricole. Si les agriculteurs sur petite exploitation peuvent être considérés comme faisant partie d'un monde populaire, la détention du capital scolaire modifie considérablement la position de ces agriculteurs, et désenclave ces milieux pour les ouvrir davantage aux autres milieux sociaux salariés. Du côté des agriculteurs les plus aisés, leur position d'héritier se trouve depuis 1992 et surtout depuis 2003 (réformes de la PAC) confortée par la rente assurée par les aides européennes. Le mouvement d'embourgeoisement agricole s'élargit à de nouvelles couches, de nouvelles productions et de nouvelles régions (Laferté, 2013). L'acquisition d'un capital scolaire a renforcé la position de cette élite économique de l'agriculture, et a permis à une grande partie des fils de petits et moyens agriculteurs restés dans l'agriculture de disposer d'un élan économique suffisant pour donner une dimension économique plus importante à leur exploitation, en même temps qu'elle a pu contribuer à l'ouverture culturelle de ces groupes. Le diplôme a donc constitué pour la génération de la première explosion scolaire, un facteur de recomposition et segmentation des groupes sociaux agricoles en même temps qu'un facteur de revalorisation des positions sociales agricoles au sein de l'échelle sociale.

\section{Les trajectoires sociales des fils qui ne deviennent pas agriculteurs}

Si les fils d'agriculteurs constituent encore la source essentielle du recrutement des futurs agriculteurs, pour la plupart des enfants d'origine agricole l'avenir le plus probable est désormais celui d'une sortie de l'agriculture. Ce phénomène s'inscrit dans un mouvement de réduction du secteur primaire et de croissance des secteurs secondaire et surtout tertiaire à partir des années 1970. C'est une "mobilité structurelle ", due à la transformation de la structure des emplois. Mais ce mouvement s'inscrit également dans une croissante "fluidité sociale " de la société française propre aux années 1960-1970 (Vallet, 1999). La mobilité sociale s'est améliorée pour ces générations et cela est à mettre en lien avec la première explosion scolaire. 


\section{Tableau d'ensemble de la mobilité hors de l'agriculture des fils d'agriculteurs}

La trajectoire des fils d'agriculteurs quarantenaires de 2003 ne differe pas fondamentalement de ce que Gollac et Laulhé avaient mis en lumière (1987, 1990). Le devenir des fils d'agriculteurs est très proche de celui des fils d'ouvriers : il est caractérisé par un accès important aux emplois salariés les plus bas de la hiérarchie sociale. Parmi les emplois dans le monde ouvrier, les fils d'agriculteurs s'orientent davantage que les fils d'ouvriers vers les emplois de type artisanaux (dans le bâtiment...), les emplois où les individus conservent une certaine autonomie (chauffeur). Ils s'orientent également davantage que les fils d'ouvriers (mais moins que la moyenne) vers des postes où une certaine compétence de métier ou de discipline scientifique est nécessaire (artisan indépendant, voire technicien ou ingénieur, professions intermédiaires commerciales ou administratives), ou vers certains postes d'employés de la fonction publique. L'accès à des emplois à dimension scientifique ou technique semble moins clivant socialement dans la mesure où les fils d'agriculteurs peuvent accéder (moyennant une formation adaptée) à des positions de ce niveau au sein des structures agricoles ou des industries agro-alimentaires. Leur bonne connaissance du secteur a pu constituer un atout pour occuper ces positions en compensant d'autres désavantages. La proximité avec les trajectoires ouvrières assoit l'image d'une population agricole qui constitue un des segments des classes populaires.

Cependant, la détention par les parents agriculteurs d'un capital économique conséquent (mesuré toujours sur la base de la dimension économique de l'exploitation, $c f$. note 4) modifie ce panorama en dessinant d'autres trajectoires : les fils d'agriculteurs de moyenne et grande exploitation sont un peu plus souvent présents que ceux de petite exploitation dans le monde indépendant $(9,6$ contre $7,2 \%)$, bien mieux représentés dans les emplois de cadre et ingénieurs $(21,1$ contre $15,5 \%)$, de professions intermédiaires $(15,4$ contre $6,3 \%)$ ou d'employés $(21,2$ contre $16,4 \%)$. À l'inverse, ils sont moins présents dans les emplois les plus bas du salariat : $19 \%$ sont ouvriers (hors agriculture) contre $43 \%$. Les différences déjà constatées en 1985 se sont creusées et le poids de l'origine sociale apparaît ici déterminant. Les fils d'agriculteurs aisés ont une trajectoire sociale proche de celle des fils d'employés. Les trajectoires des fils de petits exploitants ressemblent en revanche davantage à celles des fils d'ouvriers non qualifiés.

Les trajectoires socioprofessionnelles des fils des agriculteurs s'améliorent, même si les positions les plus élevées leur sont toujours statistiquement moins accessibles que pour d'autres PCS (professions et catégories socioprofessionnelles, nomenclature établie par l'Insee). "L'aspiration par le haut de la société " (avec la hausse du nombre d'emplois qualifiés) ainsi qu'un relatif maintien des écarts entre milieux sociaux est confirmée (Peugny, 2013). 


\section{Demeurer au sein des classes populaires}

Pour la population qui nous intéresse, l'entrée dans le salariat passe le plus souvent par une intégration au monde ouvrier (Tab. 2). Ainsi 67,6 \% des fils d'agriculteurs âgés de 40 à 49 ans qui ne sont pas devenus agriculteurs et qui sont sortis du système scolaire sans diplôme ont un emploi d'ouvrier, de même que 57,8 \% des titulaires d'un CEP et 51,8 \% des titulaires d'un CAP. Les différences en matière de diplôme sont très importantes, bien plus que l'origine sociale agricole ou ouvrière : à niveau de diplôme égal, les fils d'ouvriers et les fils d'agriculteurs ont des chances comparables d'occuper à 40-49 ans un emploi d'ouvrier.

Tab. 2. Effet du diplôme sur le devenir professionnel hors agriculture des fils d'agriculteurs âgés de 40 à 49 ans en \%(*).

\begin{tabular}{|c|c|c|c|c|c|c|}
\hline & Indépendants & $\begin{array}{l}\text { Cadres et } \\
\text { professions } \\
\text { libérales }\end{array}$ & $\begin{array}{c}\text { Professions } \\
\text { intermédiaires }\end{array}$ & Employés & Ouvriers & $\begin{array}{l}\text { Ouvriers } \\
\text { agricoles }\end{array}$ \\
\hline Aucun & 6,1 & 0,7 & 2,7 & 5,8 & 67,6 & 7,0 \\
\hline $\begin{array}{l}\text { Certificat } \\
\text { études } \\
\text { primaires }\end{array}$ & 9,8 & 1,1 & 9,2 & 12,3 & 57,8 & 5,0 \\
\hline BEPC & 8,7 & 8,3 & 25,2 & 22,2 & 29,1 & 3,0 \\
\hline CAP & 13,1 & 1,7 & 17,2 & 10,5 & 51,8 & 1,9 \\
\hline BEP & 13,2 & 5,3 & 29,1 & 10,6 & 36,1 & 3,4 \\
\hline $\begin{array}{c}\text { Bac } \\
\text { technique } \\
\text { ou brevet } \\
\text { professionnel }\end{array}$ & 10,0 & 12,8 & 47,9 & 9,5 & 12,3 & 6,2 \\
\hline Bac général & 9,6 & 18,3 & 45,2 & 16,3 & 7,7 & 0,0 \\
\hline $\begin{array}{l}\text { Université } 1^{\text {er }} \\
\text { cycle ou BTS }\end{array}$ & 10,0 & 32,7 & 51,7 & 3,3 & 1,9 & 0,0 \\
\hline $\begin{array}{l}\text { Université } 2^{\mathrm{e}} \\
\text { cycle ou plus }\end{array}$ & 5,3 & 73,4 & 14,8 & 2,4 & 3,0 & 0,0 \\
\hline Total & 10,4 & 10,1 & 21,8 & 10,1 & 40,3 & 3,3 \\
\hline
\end{tabular}

(*) La somme en ligne des pourcentages ne fait pas $100 \%$ car une modalité regroupant certains retraités et inactifs, assez peu fréquente, n'a pas été reproduite dans ce tableau.

Source : EHF1999.

Champ : individus âgés de 40 à 49 ans en 1999, fils d'un père agriculteur n'étant pas agriculteur $(N=2952)$

Lecture : 6,4 \% des fils d'agriculteurs âgés de 40 à 49 ans, sans diplôme et n'ayant pas choisi l'agriculture ont un emploi d'indépendant (artisan, commerçant, chef d'entreprise).

Test du Khi2 : significatif au seuil de 0,01\%. 
Le monde des ouvriers agricoles ${ }^{18}$ est aussi traversé par des hiérarchies internes : les fils d'agriculteurs sans diplôme ont pu y accéder plus souvent que les autres diplômés mais certains fils titulaires d'un BEP y sont également bien représentés. Dans ce secteur comme dans le reste du monde ouvrier, coexistent un salariat agricole sans qualification et un salariat agricole qualifié (notamment en viticulture ou en élevage).

L'effet du diplôme et de l'origine sociale (fils d'agriculteur sur petite/moyenne/ grande exploitation) se cumulent ou se compensent : la difficulté plus forte des fils de petit agriculteur à sortir du système scolaire avec un diplôme important les conduit alors plus fermement vers le monde ouvrier, mais le fait d'être issu d'une famille aisée peut aider à rattraper une carrière scolaire courte. Même s'il faut prendre ces chiffres avec prudence en raison des faibles effectifs des fils issus d'exploitations aisées (moyennes ou grandes), pour les fils ayant acquis au mieux un CAP, être issu d'une exploitation aisée réduit le risque d'une trajectoire vers le monde ouvrier (29,1 contre $47,4 \%$ pour ceux issus d'une petite exploitation).

Les trajectoires d'entrée dans le monde ouvrier ou employé populaire ne doivent pas être cependant considérées comme un éloignement définitif des mondes de l'agriculture (Mischi, 2013). Les doubles appartenances professionnelles existent : selon le recensement agricole 2000, 30,1\% des hommes de 40 à 49 ans travaillant sur une exploitation agricole - hors salariat - exerçaient une autre profession principale que celle d'agriculteur ; de même des participations régulières au travail agricole contribuent à faire vivre une exploitation agricole tenue par un autre membre de la famille. Ces doubles activités ouvrières et agricoles sont facilitées dans la mesure où presque les deux tiers des fils d'agriculteurs ouvriers qualifiés, et $56 \%$ des fils ouvriers non qualifiés restent dans leur région d'origine.

\section{S'intégrer aux classes moyennes}

La possession de diplômes élevés a permis d'assurer à certains enfants d'agriculteur quarantenaires comme trentenaires des positions sociales hors du monde ouvrier.

Les détenteurs de BEP ou brevet professionnel chez les quarantenaires et trentenaires, sont, on l'a vu, plus souvent que d'autres profils scolaires attirés vers l'agriculture (ou vers les emplois d'ouvriers qualifiés). Mais ils vont avoir aussi plus tendance à rejoindre les professions intermédiaires (tout particulièrement les détenteurs de bacs techniques) plus spécialement dans des postes de «techniciens » ou d' " agent de maîtrise ». Les formations de premier cycle-BTS favorisent également très fortement l'accès aux professions intermédiaires. Le diplôme semble commander, ici encore, davantage au devenir socioprofessionnel que l'origine sociale dans la mesure où les

18. Précisons que la catégorie des ouvriers agricoles est plus réduite que celle des salariés agricoles. Les salariés les plus diplômés comme les chefs de cultures par exemple relèvent donc d'une autre PCS que celle des ouvriers agricoles. 
fils d'agriculteurs et les fils d'ouvriers ont à diplôme égal des chances du même ordre d'exercer une profession intermédiaire.

Les métiers indépendants d'artisan, de commerçant ou de chef d'entreprise constituent une voie plus particulièrement prisée par les fils d'agriculteurs. Quel que soit le niveau de diplôme, ils sont toujours mieux représentés que les fils d'ouvrier. Ce milieu des indépendants est assez hétérogène scolairement puisqu'il accueille à la fois des fils d'agriculteurs promus par le système scolaire comme des fils qui n'ont pas eu accès au niveau BEP. Comme en agriculture, les capitaux économiques et relationnels sont tout aussi indispensables que le capital scolaire pour s'installer dans l'indépendance. Les trajectoires qui conduisent à l'indépendance hors de l'agriculture se font aussi souvent dans une certaine proximité géographique avec l'exploitation familiale. $71,5 \%$ des fils d'agriculteurs devenus artisans sont encore dans la région de leur enfance contre $58,2 \%$ en moyenne. Le maintien dans leur région d'enfance des fils d'agriculteurs devenus artisans ou chefs d'entreprise assure la continuité de relations sociales et familiales, sources potentielles d'aides de travail ou de soutien économique (de forme multiple, Gollac, 2008), particulièrement utiles pour la bonne marche d'une entreprise, artisanale ou autre, et pour la constitution d'une clientèle.

Disposer d'un diplôme court de l'enseignement général (le BEPC ou le bac), a favorisé plus que tout autre diplôme l'entrée des fils d'agriculteurs dans le monde des employés, principalement de la fonction publique. Les fils d'ouvriers accèdent cependant plus souvent que les fils d'agriculteurs au monde des employés alors que les seconds s'orientent davantage que les premiers vers l'indépendance professionnelle.

\section{Conclusion}

L'analyse des trajectoires scolaires et socioprofessionnelles des fils d'agriculteurs a permis de mettre au jour certains des profonds bouleversements qui ont touché le monde agricole. La première massification scolaire, les transformations des conditions d'accès aux aides économiques, les modalités de contrôle du métier, l'hétérogamie croissante dans les familles ont conduit les agriculteurs à développer un nouveau rapport à l'école. Par l'acquisition d'un capital scolaire technique (agricole) élevé, les familles ont concilié les impératifs de reproduction sociale et les exigences de construction des individus par l'école. L'acquisition du capital scolaire devient un élément central de compréhension des différentes trajectoires sociales et professionnelles des fils d'agriculteurs. Dans l'ensemble de ces trajectoires, trois grands clivages ont été mis en lumière :

Le premier clivage se situe entre ceux qui deviennent agriculteurs et ceux qui ne le deviennent pas. La génération des fils, ayant connu la première massification, qui sont devenus agriculteurs est surtout celle qui a su acquérir les diplômes techniques nécessaires (et bénéficier des aides financières liées à ces diplômes) pour s’installer. 
Ceux qui sont sortis sans diplôme du système scolaire ont davantage eu tendance à entrer dans le monde ouvrier (non agricole). La position d'agriculteur n'a plus été un choix obligé de ceux qui avaient "échoué dans les études ".

Le capital scolaire est au cœur d'un second clivage au sein de la population de ceux qui sont devenus agriculteurs. Les diplômés ont bénéficié plus systématiquement d'aides financières (DJA, aides JA) que les fils sans diplôme et ont pu donner à leur exploitation une tout autre dynamique économique. Ce premier fossé économique se double d'une différence culturelle. Les diplômés ont pu trouver des conjointes situées plus haut dans l'échelle sociale que les non-diplômés. Certes les diplômés reproduisent en partie la position économique de leurs parents en choisissant l'agriculture. Les fils de moyens et grands exploitants sont plus diplômés que les autres. Mais une bonne frange de la petite agriculture a pu également obtenir les diplômes nécessaires pour accéder aux positions élevées au sein des agriculteurs. Tous ces fils d'agriculteurs restent agriculteurs, mais la reproduction du monde agricole qu'ils sont en train d'effectuer est une reproduction dynamique tant les positions relatives à l'intérieur de ce monde changent d'une génération à l'autre. Les fils diplômés de la petite agriculture (en ascension ou pas) établissent en tout cas des passerelles avec des milieux sociaux de classes moyennes dont ils étaient auparavant distants. À l'inverse, les fils non-diplômés de la moyenne et grande agriculture ont pu reproduire la position des parents en demeurant au sein de l'univers culturel familial. En dépit d'intérêts de politique agricole partiellement communs à la profession, les distances sociales et culturelles au sein des mondes de l'agriculture sont marquées.

Le capital scolaire définit un troisième clivage parmi les fils qui ne deviennent pas agriculteurs. Les diplômés ont occupé toujours plus fréquemment des emplois de classe moyenne et dans une moindre mesure de classe supérieure. Ils sont distants socialement de ceux qui ont quitté l'école avec un bagage léger qui les conduit vers le milieu ouvrier. Cependant les ouvriers d'ascendance agricole, souvent issus de la petite agriculture, ont pu conserver des liens étroits avec leur famille et parfois une activité agricole en marge de leur travail. Les circulations entre ces deux catégories socioprofessionnelles ont été facilitées par leur proximité sociale et culturelle au sein des classes populaires.

Ainsi, avec la première explosion scolaire, la mise en place d'une formation agricole et les premières mesures de conditionnalité des aides à l'installation, les divers mondes agricoles apparaissent nettement clivés culturellement et socialement entre eux. Les effets conjoints de la politique scolaire et de la politique des structures ont éloigné de l'agriculture une part croissante des enfants d'agriculteurs qui ont pu estimer que le niveau scolaire à atteindre constituait une barrière trop élevée mais ont conduit aussi à en éloigner certains qui avaient pu acquérir une culture scolaire suffisante pour obtenir des positions sociales plus valorisantes. Le dépaysement produit par l'école a conduit à un refus plus fort du métier d'agriculteur. L'école de cette première massification, et tout particulièrement l'enseignement agricole, a par ailleurs contribué à la dépaysannisation en même temps qu’à la professionnalisation de ces nouveaux agriculteurs qui sont porteurs de valeurs bien différentes de celles auxquelles adhèrent ceux qui ont simplement hérité des pratiques et de 
l'exploitation familiales. Pour l'agriculture comme pour le monde ouvrier (Beaud et Pialoux, 2012), la scolarisation prolongée a contribué à renforcer les clivages entre membres d'un même groupe professionnel. Elle a contribué également à favoriser le rapprochement culturel des agriculteurs scolarisés avec les actifs scolarisés des autres milieux sociaux.

\section{Bibliographie}

Alarcon, L., 2008. "Maintenant, faut presque être ingénieur pour être agriculteur ", Choix et usages des formations professionnelles agricoles dans deux familles d'agriculteurs, Revue d'études en agriculture et environnement, 88, 3, 95-118.

Barthez, A., 1994. Le patrimoine foncier des agriculteurs vivant en couple, Agreste : Analyses et Études, Cahiers, 17-18, mars, 23-36.

Beaud, S., Pialoux, M., 2012. Retour sur la condition ouvrière, Paris, La découverte.

Bessière, C., 2010. De génération en génération. Arrangement de famille dans les entreprises viticoles de Cognac, Paris, Raisons d'Agir.

Champagne, P., 2002 L'héritage refusé. La crise de la reproduction sociale de la paysannerie française 1950-2000, Paris, Seuil, Points.

Chauvel, L., 2002. Le destin des générations, Paris, PUF, 2 édition.

Coulomb, P., 1990. La cogestion, une nouvelle tentative corporatiste?, in Coulomb, P., Delorme, H., Hervieu, B., Jollivet, M., Lacombe, P. (dir.), Les agriculteurs et la politique, Paris : FNSP, 147-157.

Dahache, S., 2013. La féminisation de l'enseignement agricole, sociologie des rapports de genre dans le champ des formations professionnelles, Paris, L'harmattan.

Davaillon, A., 1998. Parcours scolaires des élèves ruraux et des enfants d'agriculteurs : spécificités et évolutions, Éducation et formations, 54, déc., 97-107.

Dufour, A., Giraud, C., 2012. Le travail dans les exploitations d'élevage bovin laitier est-il toujours conjugal ? INRA Productions animales, 25, 2, juin, 169-180.

Giraud, C., 2014. Une distance sociale intime, in Boudjaaba, F. (dir.), Le travail et la famille dans le monde rural (XV'EXXIe siècles), Rennes, PUR, 185-206.

Giraud, C., Rémy, J., 2008. Les choix des conjoints en agriculture, Revue d'études en agriculture et environnement, 88, 3, 21-46.

Gollac, S., 2008. Travail indépendant et transmissions patrimoniales : le poids des inégalités au sein des fratries, Économie et statistique, 417-418, 55-75.

Gollac, M., Laulhé, P., 1987. Trois articles sur l'hérédité sociale, Économie et Statistique, 199-200, 85-90.

Hermelin-Guillou, C., 1987. L'enseignement agricole en 1987, Économie rurale, 180, 53-59.

Jégouzo, G., 1979. Le célibat paysan en 1975, Population, 1, 27-41.

Jégouzo, G., Brangeon, J.-L., 1976. Les paysans et l'école, Paris, Cujas. 
Laferté, G., 2013. L'embourgeoisement agricole dans les formes localisées de la structure sociale : revisite et ethnographie collective, Habilitation à diriger des recherches, Université Paris 4.

Maresca, S., 1988. La réussite d'une politique. Interrogations sur les effets sociaux des incitations publiques, Cahier d'économie et de sociologie rurale, 6, 5-40.

Maurin, E., 2007. La nouvelle question scolaire, Paris, Seuil, Points.

Mischi, J., 2013. Ouvriers ruraux, pouvoir local \& conflits de classe, in Campagnes populaires, campagnes bourgeoises, Agone, 51.

Euvrard, F., Rondeau, M.-C., 1985. Déroulement de la scolarité des enfants d'agriculteurs, Revue française de pédagogie, 73, oct.-déc., 7-14.

Peugny, C., 2013. Le destin au berceau, Paris, Seuil.

Poullaouec, T., 2004. Les familles ouvrières face au devenir de leurs enfants, Économie et Statistique, 371, 3-22.

Rémy, J., 2012. L'exploitation agricole : une institution en mouvement, Déméter 2013, 357-384.

Schwartz, O., 1990. Le monde privé des ouvriers, Paris, PUF.

SSP, 2011. L'agriculture en France. Premiers résultats, Présentation du 19 octobre 2011.

Vallet, L.-A., 1999. Quarante années de mobilité sociale en France. Lévolution de la fluidité sociale à la lumière de modèles récents, Revue française de sociologie, $40,1,5-64$. 